\title{
Formulating Enhancement and Restoration Strategy to Improve the Quality of Dusty Images
}

\author{
Madallah Alruwaili ${ }^{*}$,Lalit Gupta ${ }^{2}$ \\ ${ }^{1}$ Dept. of Computer Enginerring, Aljouf University, Sakaka, Aljouf 42421, KSA. \\ 2 Dept. of Electrical \& Computer Engineering, Southern Illinois University, Carbondale, IL 62903, USA. \\ * Corresponding author. Tel.: 00966503393098; email: madallah@ju.edu.sa \\ Manuscript submitted January 22, 2016; accepted March 27, 2016. \\ doi: 10.17706/jcp.12.4.291-300
}

\begin{abstract}
Analyses of images acquired in dusty environments show that the images tend to have noise, blur, a small dynamic range, low contrast, diminished blue components, and high red components. The goals of this paper are to develop a modeling of dust noise on ordinary images and formulating enhancement and restoration strategy to improve the quality of dusty images using a sequence of image processing steps. This paper proposes that the dust noise model on ordinary images consisting of the atmospheric turbulence-blurring model, adjusting image brightness, and simulating real dust images colors contrasts. In addition, an automatic color correction algorithm consisting of image restoration using the Wiener filter, luminance stretching using $\mathrm{YCbCr}$ color model, and enhancing the image contrast using a modified homomorphic filter. Enhancement experiments are conducted on real and simulated dusty images and it is shown that the strategy is quite effective in enhancing dusty images. Furthermore the results are superior to those obtained through, a statistical adaptive algorithm, histogram equalization, gray world, and white patch algorithms. In addition, the complexity of the proposed algorithm is very low thus making it attractive for real time-image processing.
\end{abstract}

Key words: Image enhancement, image restoration, dust images, and color balance.

\section{Dust Images}

Dust storms occur in many regions of the world, especially in the Middle East. Due to poor visibility, daily life comes to a complete stop. Commerce and video surveillance are adversely effected. Safety is compromised, especially during driving. A possible solution to this frequent and serious problem is to acquire images during dust storms and develop methods to "see through" the dust to improve visibility. Automobiles equipped with such a system will be able to assist drivers to navigate safely.

However, images acquired during a dust storm have very poor quality. Typically, the images tend to be have noise, blur, a small dynamic range, low contrast, diminished blue components, and high red components. The poor quality of the image is primarily due to light scattering, which is produced by reflectance and deflection of light incident on the dust particles. The color is changed by the attenuation of different wavelengths. Additionally, the image has blur due to the motion of the dust particles.

\section{Color Constancy and Correction}

Color constancy is the ability of the human visual system to perceive color correctly in varying 
illuminations [1]. The main goal of color constancy algorithms is to enhance an image such that it matches an image taken under canonical light sources.

The Retinex Theory has been used to solve color constancy problems through a very simplified but effective model of the human visual system [2]. The word 'retinex' is derived from two words 'retina' and 'cortex' advocating that both the eye and the brain are involved in the processing. The white patch algorithm is a simplified approach of the Retinex theory [2], [3]. It assumes that there is white patch in an image and looks for this patch in a given image. The white patch algorithm is evaluated as the maximum intensity of the $\mathrm{R}, \mathrm{G}$, and B color components.

Another well-known algorithm for color correction is the gray world algorithm formulated by Buchsbaum [4]. Numerous algorithms are based on the gray world assumption, [5], [6]. The gray world assumes that an image, on average, is a neutral gray. Assuming that there is a good distribution of colors in our scene, the average reflected color is assumed to be the color of the light. Therefore, the illumination color-cast can be estimated by comparing the average color to gray [4]. This algorithm produces good results for images having good distribution of colors. However, it does not produce good results for images, which have a dominant color [7], [8]. Many researchers have used histogram equalization for color correction. Histogram equalization aims to obtain an enhanced image, which has a uniformly distributed histogram. The operation of histogram equalization is implemented by remapping the pixels values of the image using the probability distribution of the input pixels values. It flattens and expends the dynamic range of the histogram and produces an overall enhanced image [9]. Employing a global approach of histogram equalization is good for overall contrast enhancement, however, the drawbacks of this approach are that unrealistic visual appearances may occur when the output image is not fully equalized. Better results can be obtained by processing the color components in the $\mathrm{YCbCr}$ or the HSI color model spaces [10], [11]. The Weiner filter has been shown to be quite effective in removing blur from dusty images [12].

\section{The Dust Noise Model}

The first step in removing the effects of dust weather is to understand the change that occurs in a dust image compared to a general image. In addition, generating a dust image is necessary to solve problem of limiting a data set. Therefore, this part proposes a new noise model to generate dust on general images. The proposed dust noise model consists of the following three steps: adding blur to RGB color components, changing image brightness by using gamma, and degrading image colors contrasts.

\subsection{Blurring Noise}

Noise is basically unwanted information that degrades the image quality. In digital images, the main source of noise arises through image acquisition or through image transmission. Dust weather negatively affects the performance of image sensors. As a result, captured images have blur noise. Therefore, generating blur noise to an image is essential in a dust noise model. Here, the atmospheric turbulence blurring model proposed by Hufnagel and Stanley is used [13]. We model the blur image as the linear convolution of each color component $\mathrm{R}, \mathrm{G}$, and $\mathrm{B}$ and the point spread function associated with the atmospheric turbulence-blurring model. The following formula is used for generating blur noise

$$
H(u, v)=e^{-k\left(u^{2}+v^{2}\right)^{\frac{5}{6}}}
$$

where $u$ and $v$ are the horizontal and vertical frequency variables respectively. $k$, controls the severity of the blur. If the value of $k$ is large, that means the severity of the blur is very strong whereas if the value of $k$ is very low, it means that the turbulence is not that strong. Here, $\mathrm{k}$ value is determined to be 0.0025 in order to simulate a severe turbulence. 


\subsection{Image Brightness}

Poor environmental conditions in the captured scene, such as dust, affect the brightness quality of the images. The second step of the proposed dust noise model is simulating image brightness. Brightness is an attribute of visual perception in which a source seems to be radiating or reflecting light. In a digital image, brightness describes the range from black to white, and a certain amount of brightness is crucial for human eyes to make a color pixel visible. In the RGB color model, brightness can be thought of as the mean $\mu$ of the red, green, and blue color components.

$$
\mu=\frac{\mathrm{R}+\mathrm{G}+\mathrm{B}}{3}
$$

Here, gamma correction is used to model dust image brightness. Gamma correction uses a non-linear operation to adjust darkness or lightness of an image [14]. Gamma correction is expressed as

$$
\mathrm{V} \text { out }=\mathrm{V} \text { in } \gamma
$$

where $V$ out is the output brightness of color component value, $V$ in is the input brightness of color component value and, $\gamma$ represents gamma value. In general, gamma value can be from 0 to 10 . If the gamma value is less than 1 , the image becomes darker. If the gamma value is greater than 1 , the image becomes lighter. A gamma value equal to 1 means no change occurs in image brightness. In the proposed dust noise model, gamma parameter is calculated directly from the color components without prior knowledge as follows:

$$
\gamma=\frac{(\operatorname{MaxR}+\operatorname{MaxB}+\mathrm{MaxG})}{3}
$$

MaxR is the maximum value of red color component, MaxG is the maximum value of green color component and, MaxB is the maximum value of blue color component. Here, gamma value is the average of all maximum values for better brightness degrading. After calculating gamma value, gamma correction is applied to degrade each color component R, G, and B separately.

\subsection{Image Color Contrast}

The last step of the proposed dust noise model is simulation of dust image colors contrasts. Contrast is defined as the separation between the darkest and brightest intensities of the image. If the distribution of the image intensities has a small spread, then the image has a low contrast, while intensities with a wide spread have a high contrast. In visual perception, contrast is the difference in color that makes an object distinguishable. Red, green, and blue contrasts interact together and produce the overall image. Here, the proposed dust noise model adjusts independently the R, G, and B contrasts in order to generate a dust image. The proposed noise model is designed based on a statistical analysis of real dust images histograms.

The red color component is the heaviest and the dominant color. The contrast of red color component is very bright. Therefore, the following equation is used toward the contrast of red color component to the maximum value, which is 255 .

$$
\mathrm{R}_{*}=\left(\mathrm{R}_{\mathrm{c}}-\mathrm{MinR}\right) \frac{\text { MaxR }}{(\text { MaxR-MinR })}
$$

$R_{*}$ is output pixel value and $R_{c}$ is current pixel value. $\operatorname{Max} R$ and $\operatorname{Min} R$ are maximum and minimum pixels values in red color component respectively.

In a dust image, the pixel values of the green color component are approximately in the middle side of the intensity range. In addition, the histogram of the green color component represents a low-contrast image. The following equation is used toward the contrast of the green color component to be in the middle side of the intensity range.

$$
\mathrm{G}_{*}=\left(\mathrm{G}_{\mathrm{c}}-\mathrm{MinG}\right) \frac{\text { MeanG }}{(\text { MaxG-MinG })}
$$


$G_{*}$ is the output pixel value and $G_{c}$ is the current pixel value. $\operatorname{Max} G, \operatorname{Min} G$, and Mean $G$ are maximum, minimum, and mean pixel values of the green color component, respectively.

The blue color component is normally the lowest color value compare to red and green values. The contrast of blue color component is considered dark and poor. The next equation is used in order to degrade and shift the contrast of green color component to the left side of the intensity range.

$$
\mathrm{B}_{*}=\left(\mathrm{B}_{\mathrm{c}}-\mathrm{BinG}\right) \frac{\text { MeanB } / 2}{(\text { MaxB-MinB })}
$$

$B_{*}$ is the output pixel value and $B_{c}$ is the current pixel value. $\operatorname{Max} B, \operatorname{Min} B$, and $\operatorname{Mean} B$ are maximum, minimum, and mean pixel values of blue color component, respectively.

\section{An Automatic Color Correction Algorithm}

The proposed algorithm is based on the Wiener filter, luminance stretching, and modified homomorphic filter. The first step of the proposed algorithm is applying the Wiener filter to restore image pixels and remove the blur noise. The second step converts the RGB color model to the YCbCr color model. In the $\mathrm{YCbCr}$ color model, the luminance is stretched to adjust the illumination of the image. Consequently, adjusting image illumination maintains the whole image's luminance at the same level automatically to achieve high quality. The third step is converting the $\mathrm{YCbCr}$ color model to the RGB color model. The last step applies a modified homomorphic filter to enhance an image contrast in order to get true colors. Each step is discussed in detail in the following sections of the paper.

\subsection{The Wiener Filter}

The Wiener filter is a beneficial method to decrease or remove blurring noise from dust images. The Wiener filter minimizes the mean square error between the degraded image and the desired image [15]. The restored image $\hat{F}(u, v)$ is given by:

$$
\widehat{\mathrm{F}}(u, v)=\left[\frac{1}{H(u, v)} \frac{|H(u, v)|^{2}}{|H(u, v)|^{2}+K}\right] G(u, v)
$$

where $G(u, v)$ is the degraded input image. The value of $K$ is chosen interactively to yield the best visual result.

\subsection{Luminance Stretching}

The second step of this proposed algorithm is luminance stretching. Luminance explains the range from black to white, and a certain amount of luminance is crucial for the human eyes to make a color pixel visible. Luminance is a measurement used to describe the perceived brightness of a color. Therefore, adjusting the luminance in dust images is important in order to achieve good enhancement. The proposed algorithm enhances the entire image's illumination at the same level to achieve high-quality images. The proposed algorithm uses the $\mathrm{YCbCr}$ color model in order to stretch the image illumination. The YCbCr color model was designed to separate chrominance from luminance. $Y$ is the luminance component and $\mathrm{Cb}$ and $\mathrm{Cr}$ are the chrominance components. The $\mathrm{YCbCr}$ color model is easier and faster to be calculated among the other color models. Luminance is dependent on the $R, G$ and $B$ color components. Each pixel value in all color components is needed to calculate the image luminance. The equations below are used to convert the RGB color model to the YCbCr color model:

$$
\begin{gathered}
Y=0.299 * R+0.587 * G+0.114 * B \\
\mathrm{Cb}=-0.299 * R-0.587 * G+0.886 * B=B-Y \\
\mathrm{Cr}=0.701 * R-0.587 * G-0.114 * B=R-Y
\end{gathered}
$$

After converting the image to the $\mathrm{YCbCr}$ color model, the luminance component is used to improve the 
image quality while no change occurs for the other components in order to avoid creating new colors. The proposed algorithm stretches the luminance toward both directions 0 and 255, which helps to increase the histogram and become a fully stretched. Using the luminance parameter is crucial in helping to obtain the true illumination of the dust image. The following formula is used for luminance stretching [16].

$$
P_{\mathrm{o}}=\frac{\left(P_{i}-\min \right) \times 255}{(\max -\min )}
$$

where $P_{\mathrm{o}}$ is the stretched pixel value, $P_{i}$ is the current pixel value, min is the minimum pixel value of the original luminance, and max is the maximum pixel value of the original luminance.

The next step of the proposed algorithm is to convert the image from the YCbCr color model to the RGB color model. The following equations are applied to each pixel of the image to get the true pixel value in the RGB color model.

$$
\begin{gathered}
R=Y+\mathrm{Cr} \\
G=Y-0.194 * \mathrm{Cb}-0.509 * \mathrm{Cr} \\
B=Y+C b
\end{gathered}
$$

\subsection{Modified Homomorphic Filtering}

Homomorphic filtering is a generalized method for image enhancement [15], [17]. It is filtering method that enables control of both the high frequency and low frequency components. Variations of homomorphic filtering have been used for color and illumination correction [18]. An image consists of two main components, illumination and reflectance. The illumination component results from lighting conditions present when the image is taken [15]. The reflectance component results from the way the objects in the image reflect light. Homomorphic filtering is the frequency domain filtering that enhances the image brightness and contrast. The image model for homomorphic filter can be written as follows:

$$
f(x, y)=i(x, y) \times r(x, y)
$$

where $i(x, y)$ represents illumination and $r(x, y)$ represents reflectance. The homomorphic filtering contains five-step process and these steps are applied independently for each R, G and B color component.

To apply the homomorphic filter, the natural logarithm transform is taken for both sides to decouple the $i(x, y)$ and $r(x, y)$ components

$$
z(x, y)=\operatorname{In} f(x, y)=\operatorname{In} i(x, y)+\operatorname{In} r(x, y)
$$

Next, transforming an image into the frequency domain uses the Fourier transform.

$$
Z(u, v)=F i(u, v)+F r(u, v)
$$

where $\operatorname{Fi}(u, v)$ is the Fourier transforms of $\operatorname{In} i(x, y)$ and $\operatorname{Fr}(u, v)$ is the Fourier transforms of $\operatorname{In} r(x, y)$. Next is the application of a filter function $H(u, v)$ to $Z(u, v)$ in the frequency domain.

$$
\begin{gathered}
S(u, v)=H(u, v) \times Z(u, v) \\
S(u, v)=H(u, v) \times F i(u, v)+H(u, v) \times F r(u, v)
\end{gathered}
$$

where $S(u, v)$ is the Fourier transform of the result. Here, to enhance the dust images, a modified Butterworth high pass (MBHP) filter is used. The MBHP is used to address the problem of enhancing the quality of a highly degraded image. In dust situations, all color components have contrast and color problems. Therefore, using a MBHP filter to enhance R, G, and B color components is essential. The MBHP filter can enhance the appearance of an image by simultaneous dynamic range compression and contrast enhancement. The MBHP filter function can be shown below. 


$$
H(u, v)=\left(\gamma_{H}-\gamma_{L}\right)\left[\frac{1}{1+\left[D_{0} / D(u, v)\right]^{2 n}}\right]+\gamma_{L}
$$

where $\gamma_{H}$ is the high frequency gain and $\gamma_{L}$ is the low frequency gain. In addition, $\mathrm{n}$ expresses the order of the filter. $D_{0}$ is the cutoff distance from the center and $D(u . v)$ is given by:

$$
\mathrm{D}(\mathrm{u}, \mathrm{v})=\left[\left(\mathrm{u}-\frac{\mathrm{M}}{2}\right)^{2}+\left(\mathrm{v}-\frac{\mathrm{N}}{2}\right)^{2}\right]^{1 / 2}
$$

where $M$ is the number of rows and $\mathrm{N}$ is the number of columns of the original image. Empirically, when applying the MBHP process onto different dust images, the best result has been found when $\gamma_{H}=0.7, \gamma_{L}=$ $0.1, n=2$, and $D_{0}=10$. Consequently, apply the inverse Fourier transform to get the enhanced image in the spatial domain.

$$
\begin{gathered}
S(x, y)=\mathcal{F}^{-1}\{S(u, v)\} \\
S(x, y)=\mathcal{F}^{-1}\{H(u, v) \times F i(u, v)+H(u, v) \times F r(u, v)
\end{gathered}
$$

In the final step the enhanced image $g(x, y)$ is obtained by using the following equation:

$$
g(x, y)=e^{s(x, y)}
$$

\section{Experiment and Evalution}

The dust noise model was developed in this paper using Matlab 2013. Examples of generated dust images are shown in Fig. 1. Results show that this model has the ability to simulate a real dust image. It is clearly that generated images have a blur noise, poor illumination and colors. Therefore, images generated by using the dust noise model are close to real dust images.

The data results were evaluated based on subjective and objective methods. The first performance measurement was made subjectively by using human perception. The second performance measurement was made by using quality assessment methods. It is based on well-defined mathematical models that can predict perceived image quality between a noisy image and an original image. This paper used three methods, namely root mean square error (RMSE), peak signal to noise ratio (PSNR), and structural similarity index (SSIM).

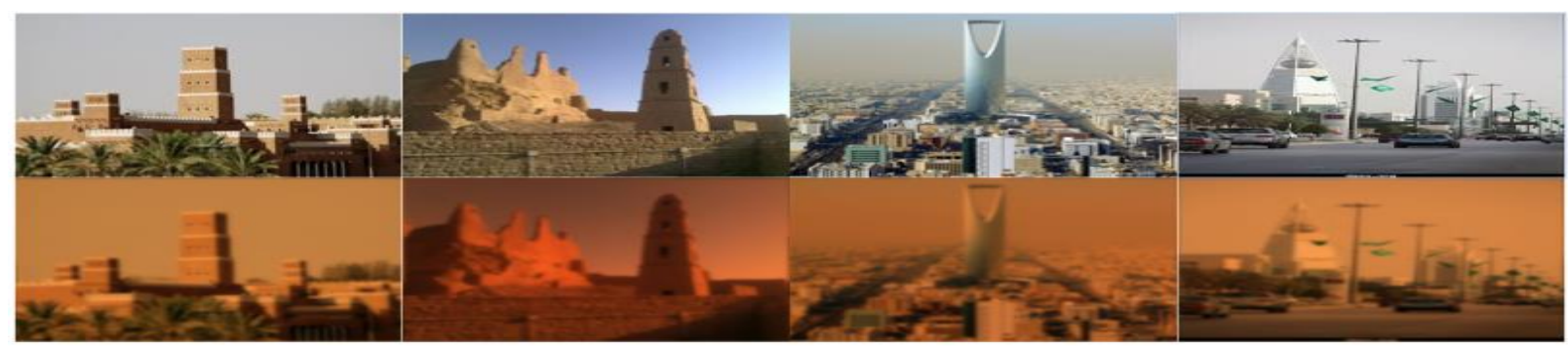

Fig. 1. Examples of images before and after applying the dust noise model.

\subsection{Human Perception}

Subjective evaluations are usually conducted in studies involving the enhancement of images [19]. In this study, the results were evaluated subjectively by a group of 30 participants analysing 50 dust images. The participants compared the results of the proposed algorithm (D) with four well-known existing color-correction methods: gray world algorithm (G), white patch algorithm (W), and histogram equalization (H) and a statistical adaptive algorithm (S). For each participant, nine of the 50 original images were randomly chosen to evaluate the performance. Each processed image was ranked in terms of the clarity, blur removal, and color balance. Examples of dust images and processed images are shown in Fig. 2. It is clear that most of participants strongly agree that images produced using our algorithm (D) has the best clarity and the least dominate color, blur, and noise. 


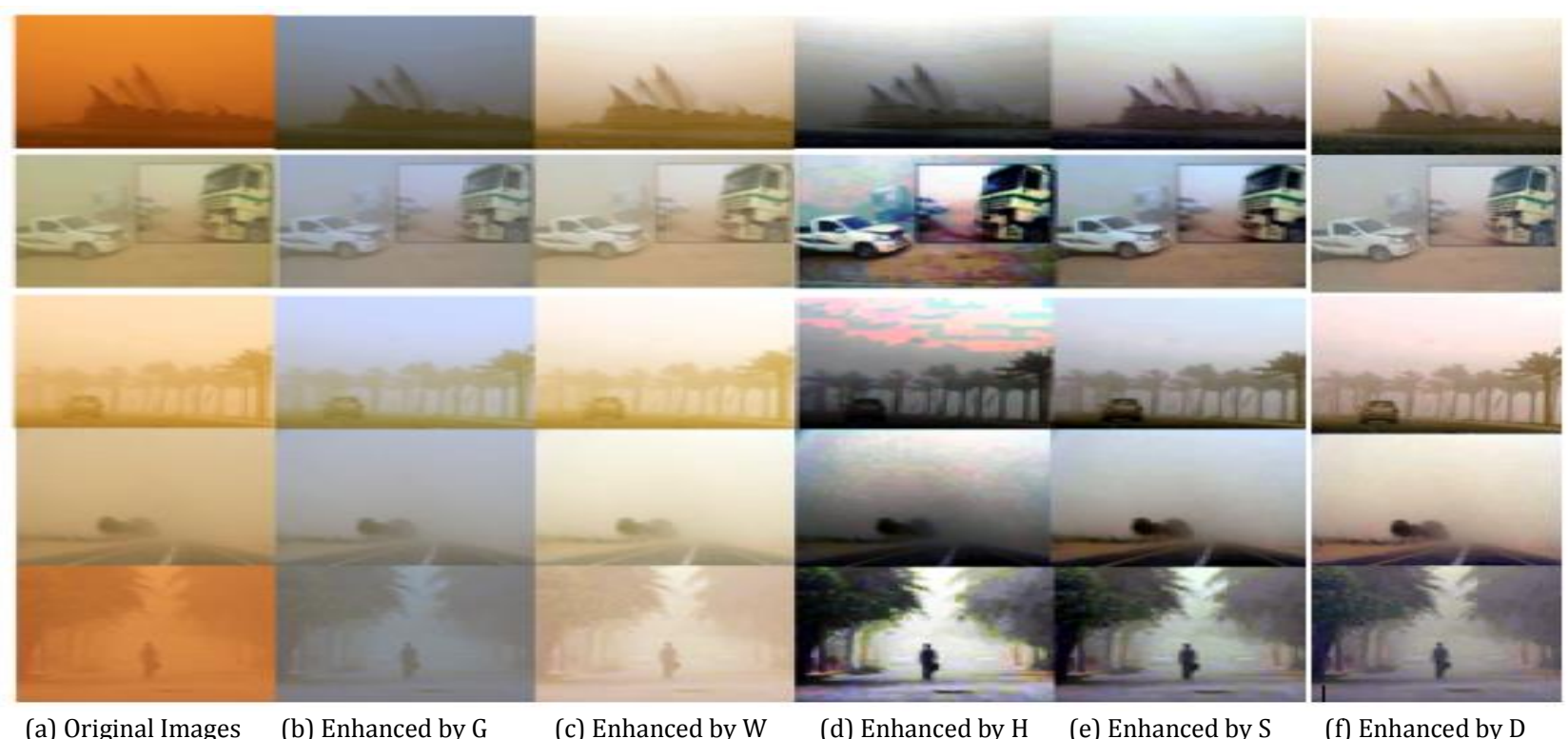

Fig. 2. Examples of images before and after enhancement.

\subsection{The Root Mean Square Error (RMSE)}

The use of the RMSE is common, and many researchers have used it for result evaluation. The root mean square error RMSE is the cumulative squared error between a noisy and an original image. The equation below used to find RMSE.

$$
R M S E=\sqrt{\frac{1}{M N} \sum_{i=1}^{M} \sum_{j=1}^{N}[O(i, j)-D(i, j)]^{2}}
$$

When the RMSE is decreased to zero, the pixel-by-pixel matching of the image becomes perfect. If the RMSE is small enough, it corresponds to a high quality restored and enhanced image. Also, in general, the RMSE value increases as the noise increases.

The evaluation based on the average RMSE value of 50 images shows that both the automatic color correction algorithm (D) and the statistical adaptive algorithm (S) have smaller average values compared to other algorithms, as shown in Table 1. Table 1 below clearly shows that our algorithms S and D produced better enhanced and restored images compared to the other algorithms.

\subsection{Peak Signal to Noise Ratio (PSNR)}

The second objective method is peak signal to noise ratio or PSNR. PSNR is a common method used to measure the quality of a restored image. The signal in this case is the original image, and the noise is the restored image that was introduced by the enhancement and restoration algorithm. A high PSNR indicates that the restored and enhanced image is high quality because it means that the ratio of the original image to the restored image is higher.

In order to calculate PSNR, we need to calculate the mean square error (MSE) between the original and the restored image. The mathematical formulas for the two are:

$$
\begin{gathered}
M S E=\frac{1}{M N} \sum_{i=1}^{M} \sum_{j=1}^{N}[O(i, j)-D(i, j)]^{2} \\
P S N R=10 * \log 10\left(\frac{255^{2}}{M S E}\right)
\end{gathered}
$$

Here, this method was applied to measure the enhanced and restored images and compared to the original images. The average value of PSNR of the 50 images shows that both the automatic color correction $\mathrm{D}$ and the statistical adaptive algorithm $\mathrm{S}$ have higher average values compared to the other algorithms, as 
shown in Table 1. Automatic color correction $\mathrm{D}$ is the best and the histogram equalization is the worst algorithm.

\subsection{The Structural Similarity Index (SSIM)}

The third objective method is the structural similarity index (SSIM). SSIM is a recently proposed method for measuring the similarity between two images. SSIM was proposed to improve on traditional metrics, such as MSE and PSNR, which have proven to be inconsistent with perception of a human eye. It is an image quality metric, which assesses the visual impact of three characteristics of an image: luminance, contrast and structure. The SSIM value lies between $[0,1]$. The overall index is a multiplicative combination of the three terms as shown in the following formula:

$$
\operatorname{SSIM}(x, y)=[L(x, y)]^{\alpha} . .[C(x, y)]^{\beta}[s(x, y)]^{\gamma}
$$

where $\alpha, \beta$ and $\gamma$ are parameters that can be used to modify the relative importance of the three components[20], [21]. The next equations show how SSIM is calculated:

$$
\begin{gathered}
L(x, y)=\frac{2 \mu_{x} \mu_{y}+C_{1}}{\mu_{x}^{2}+\mu_{y}^{2}+C_{1}} \\
C(x, y)=\frac{2 \sigma_{x} \sigma_{y}+C_{2}}{\sigma_{x}^{2}+\sigma_{y}^{2}+C_{2}} \\
S(x, y)=\frac{\sigma_{x y}+C_{3}}{\sigma_{x} \sigma_{Y}+C_{3}} \\
\operatorname{SSIM}(x, y)=\frac{\left(2 \mu_{x} \mu_{y}+C_{1}\right)\left(2 \sigma_{x y}+C_{2}\right)}{\left(\mu_{x}^{2}+\mu_{y}^{2}+C_{1}\right)\left(\sigma_{x}^{2}+\sigma_{y}^{2}+C_{2}\right)}
\end{gathered}
$$

where $\mu x, \mu y, \sigma x, \sigma y$, and $\sigma x y$ are the local means, standard deviations, and cross-covariance for images $x$, y. $C 1=(0.01 * L) .^{\wedge} 2$, where $L$ is the specified dynamic range value.

$$
\begin{gathered}
C 2=(0.03 * L) \cdot{ }^{\wedge} 2 \\
C 3=C 2 / 2
\end{gathered}
$$

The average SSIM values of the 50 images that were produced by using each of the above algorithms were calculated. The best result was obtained by using the automatic color correction (D), which was 0.988.The second best result was obtained by using the statistical adaptive algorithms (S), which was 0.932 . Table 1 shows the average SSIM value of the 50 images.

Table 1. The Average Value of RMSE, PSNR, and SSIM of 50 Images

\begin{tabular}{|c|c|c|c|c|c|c|}
\hline $\begin{array}{c}50 \text { Images } \\
\text { average value of }\end{array}$ & $\mathrm{N}$ & $\mathrm{S}$ & $\mathrm{D}$ & $\mathrm{G}$ & $\mathrm{W}$ & $\mathrm{H}$ \\
\hline RMSE & 71.8 & 20.9 & 9.3 & 57.7 & 23.3 & 38 \\
\hline PSNR & 25.84 & 31.56 & 41.77 & 25.52 & 30.06 & 24.43 \\
\hline SSIM & 0.837 & 0.932 & 0.988 & 0.834 & 0.869 & 0.816 \\
\hline
\end{tabular}

\section{Conclusion}

This paper proposed the dust noise model that consists of three main steps. In the first step, the atmospheric turbulence-blurring model proposed by Hufnagel and Stanley was used to generate a blur noise. A constant value was used in order to simulate severe turbulence. In the second step, the gamma correction was used to model dust image brightness. The gamma parameter was calculated directly from the color components without prior knowledge. The third step simulated real dust image colors contrasts. The noise model was designed based on a statistical analysis of real dust image histograms. It was an 
adaptive model that extracted necessary parameters directly from the original images without prior knowledge in order to degrade each color contrast independently. As a result, the dust noise model could simulate a real dust image.

The second part of this paper developed the automatic color correction algorithm (D) for dust image enhancement and restoration. This algorithm was based on the Wiener filter, luminance stretching, and the modified homomorphic filter. The Wiener filter was used to restore image pixels and remove the blur noise. An image was converted from the RGB color model to the $\mathrm{YCbCr}$ color model. In the YCbCr color model, the luminance was stretched to adjust the illumination of the image at the same level automatically to achieve high quality. In the RGB color model, the modified homomorphic filter was applied to enhance the image contrast in order to get true colors.

The results of proposed algorithm expressed the ability to solve difficult problems with blurring images, limited range, low contrast, non-uniform lighting, diminished blue color and dominant red color. The proposed algorithm took care of the image's properties in order to enhance the image based on its characteristics. The proposed algorithm was evaluated using the four well-known methods: human perception, root mean square error, peak signal to noise ratio, and structural similarity index. The use of these methods ensures that the proposed algorithms were thoroughly evaluated. The proposed algorithm was compared with four well-known existing algorithms namely histogram equalization $(H)$, gray world $(G)$, white patch (W), and the statistical adaptive algorithm. The results show that the proposed algorithm has performed well, and produced better results compared to existing algorithms.

\section{References}

[1] Chakrabarti, A., Keigo, H., \& Todd Z. (2012). Color constancy with spatio-spectral statistics. IEEE Transactions on Pattern Analysis and Machine Intelligence, 34(8), 1509-1519.

[2] Land, E. H., \& McCann, J. J. (1971). Lightness and retinex theory. Journal of the Optical Society of America A, 61, 1-11.

[3] Cardei, V. C., \& Funt, B. (1999). Committee-based color constancy. Proceedings of the IS\&T/SID Seventh Color Imaging Conference on Color Science, Systems and Applications (pp. 311-313). Scottsdale, Arizona.

[4] Buchsbaum, G. (July 1980). A spatial processor model for object colour perception. Journal of the Franklin Institute, 310(1), 1-26.

[5] Moore, A., Allman, J., \& Goodman, R. M. (1991). A real-time neural system for color constancy. IEEE Transactions on Neural Networks, 2(2), 237-247.

[6] Tominaga, S. (1991). Surface identification using the dichromatic reflection model. IEEE Transactions on Pattern Analysis and Machine Intelligence, 13(7), 658-670.

[7] Bianco, S., Gasparini, F., \& Schettini, R. (2007). Combining strategies for white balance. Proceedings of SPIE: Vol. 6502 (pp. 175-178).

[8] Weng, C., Homer, C., \& Fuh, C. (2005). A novel automatic white balance method for digital still cameras. IEEE International Symposium on Circuits and Systems: Vol. 4 (pp. 3801-3804).

[9] Cheng, Y. C., Chan, W. H., \& Chen, Y. Q. (1995). Automatic white balance for digital still camera. IEEE Transactions on Consumer Electronics, 41, 460-466.

[10] Chiou, T. S., Fuh, C. S., \& Chikane, V. (2002). Automatic white balance for digital still camera. Proceedings of Conference on Artificial Intelligence and Applications (pp. 475-480) Tai-Chung, Taiwan.

[11] Kao, W. C., Wang, S. H., Kao, C. C., Huang, C. W., \& Lin, S. Y. (2006). Color reproduction for digital imaging system. Proceedings of IEEE International Symposium on Circuits and Systems.

[12] Alruwaili, M., \& Gupta, L. (2015). A statistical adaptive algorithm for dust image enhancement and restoration. Proceedings of 2015 IEEE International Conference on Electro/Information Technology (pp. 
286-289).

[13] Hufnagel, R. E., \& Stanley, N. R. (1964). Modulation transfer function associated with image transmission through turbulence media. Optical Society of America Journal A, 54, 52-61.

[14] Chiu, Y. S., Cheng, F. C., \& Huang, S. C. (Oct. 2011). Efficient contrast enhancement Using adaptive gamma correction and cumulative intensity distribution. Proceedings of IEEE Conf. Syst. Man Cybern (pp. 2946-2950).

[15] Gonzalez, R. C., \& Woods, R. E. (2008). Digital Image Processing. New Jersey: Pearson Prentice Hall.

[16] Fisher, R., Perkins, S., Walker, A., \& Wolfart, E. (2008). Contrast stretching Retrieved 25th December 2013, from Web site: http://homepages.inf.ed.ac.uk/rbf/HIPR2/stretch.htm

[17] Hadhoud, M. M. (1999). Image contrast enhancement using homomorphic processing and adaptive filters. Proceedings of Radio Science Conference (pp. 89-92). Cairo.

[18] Voicu, L. I., Harley, R. M., \& Weeks, A. R. (1997). Practical consideration on color image enhancement using homomorphic filtering. Journal of Electronic Imaging, 61, 108-113.

[19] Barnard, K., Cardei, V., \& Funt, B. (2002). A comparison of computational color constancy algorithms-part I: Experiments with image data. IEEE Transactions on Image Processing, 11(9), 2002.

[20] Wang, Z., Bovik, A. C., Sheikh, H. R., \& Simoncelli, E. P. (Apr. 2004). Image quality assessment: From error visibility to structural similarity. IEEE Transactions on Image Processing, 13(4), 600-612.

[21] Loza, et al. (2006). Structural similarity-based object tracking in video sequences. Proceedings of the 9th International Conf. on Information Fusion.

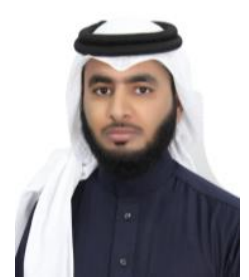

Madallah Alruwaili was born in Aljouf, KSA, in 1983. He received the B.E. (Hons) degree in computer science from the Aljouf University, KSA, in 2005, the M.S. degree in computer science from University of Science, Malaysia, in 2009, and the Ph.D degree in electrical and computer engineering from Southern Illinois University, Carbondale, Illinois, USA, in 2015. He is currently an Assistant Professor of Computer Engineering and Networks at Aljouf University, Sakaka, Aljouf, KSA. He is now a vice dean of College of Computer and Information Sciences. His research interests include image processing, image quality analysis, pattern recognition, computer vision, and biomedical imaging.

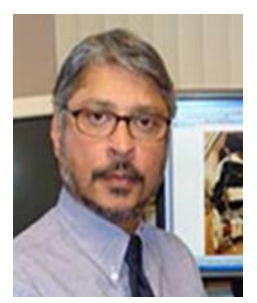

Lalit Gupta received the B.E. (Hons) degree in electrical engineering from the Birla Institute of Technology and Science, Pilani, India in 1976, the M.S. degree in digital systems from Brunel University, Middlesex, England in 1981, and the Ph.D degree in electrical engineering from Southern Methodist University, Dallas, Texas in 1986. He is currently a Professor of electrical and computer engineering at Southern Illinois University, Carbondale, Illinois. He also serves as the Associate Chair and Graduate Program Director. His research interests are in pattern recognition, data and information fusion, image processing, neuroinformatics, and neural networks. He has been awarded research contracts/grants from the Army Research Office, Seagate Technology, Cleveland Medical Devices/NIH, Think-A-Move Inc./NIH, Neuronetrix/NIH, Naval Postgraduate School, Rockwell-Collins, and CTB/McGraw-Hill. Dr. Gupta has numerous publications in the areas of evoked potential analyses and classification, multi-channel/sensor information fusion strategies, and neural networks. 